\title{
Esthetic Perception of Various Forms of Anterior Diastemas during Smiling
}

\author{
Divya Singla ${ }^{1} \quad$ Neha Stan ${ }^{1}$ Sanjay Mittal ${ }^{1} \quad$ Mandeep Bhullar ${ }^{1} \quad$ Isha Aggarwal ${ }^{1} \quad$ Rameez Hassan $^{1}$
}

${ }^{1}$ Department of Orthodontics and Dentofacial Orthopedics, Bhojia
Dental College and Hospital, Baddi, Solan, Himachal Pradesh, India

\begin{abstract}
Address for correspondence Neha Stan, MDS, Department of Orthodontics and Dentofacial Orthopaedics, Bhojia Dental College and Hospital, Baddi, Solan, Himachal Pradesh, India (e-mail: nu.wondr8@gmail.com).
\end{abstract}

\begin{abstract}
Introduction Midline diastema is a common esthetic problem in mixed and permanent dentition. It is considered attractive in some cultures and unattractive in others. Aim The aim of this study is to compare the perceptions of anterior diastema by dental students and laypersons.

Materials and Methods The study was conducted on 100 undergraduate students of Bhojia Dental College $(n=50)$ and Bhojia Nursing College $(n=50)$, Himachal Pradesh, India. A self-prepared questionnaire was given, which consisted of two parts: first part had personal details (name, age, sex, knowledge of diastema) and the second part contained an edited photograph of a patient who had reported to the Department of Orthodontics, Bhojia Dental College, with the chief complaint of midline diastema. The photographs were modified by photoediting software and arranged according to the previous studies on anterior diastema by Frush and Fisher, Lombardi, and Simian. A visual analog scale with scores $1-4$ (1, most attractive; 2 , attractive; 3 , less attractive; 4 , least attractive) was used to rank these photographs by the students. Comparison between various variables was made by using chi-squared test.

Keywords

- midline diastema

Results Smile attractiveness from the most to the least attractive was Simian > Frush and Fisher > Lombardi > midline diastema among both dental and nursing students. Gender and presence of diastema had no relation with the students' perceptions $(p>0.05)$.

- perception

- spacing

- attractiveness

Conclusion The location and width of diastema had an important role in the perception of dental diastemas. Midline diastema was a gap with most negative perception.
\end{abstract}

\section{Introduction}

Mouth is the most potent factor in making or marring the beauty of the face. In social interaction, our attention is mainly directed toward the mouth and eyes of the face of the person speaking. ${ }^{1}$ People's concern about the beauty and attractiveness of their teeth began more than 2,000 years ago. ${ }^{2}$

In the anterior region, diastemas affect smile esthetics. Midline diastemas are considered as unsightly. Broadbent ${ }^{3}$ referred to transient midline diastemas during childhood as an "ugly duckling" stage. However, in the 1960s and 1970s diastema was considered a sign of beauty in many traditional
African cultures, but, nowadays, most people seek dental treatment for diastema closure. ${ }^{4}$

Midline diastema is a space (or gap) between the maxillary central incisors. The space can be a normal growth characteristic during the primary and mixed dentition and generally is closed by the time the maxillary canines erupt. ${ }^{5}$ There are wide variations of perceptions of midline diastema among different cultures. The teeth on either side of a space are called "dents du bonheur" or "lucky teeth" in France. ${ }^{6}$ Diastema in dentistry has been defined as a space $>0.5 \mathrm{~mm}$ between the proximal surfaces of adjacent teeth. True midline diastema was defined as one without periodontal/
License terms

()(1) $\odot \circledast$ 
periapical involvement and with the presence of all anterior teeth in the arch. $^{7}$

The prevalence of median diastema is high in children, decreases dramatically between 9 and 11 years of age, and continues a gradual decrease up to 15 years of age. Maxillary midline spacing is common when compared with mandibular. Females exhibit a greater prevalence. ${ }^{8}$

The founding fathers of dental esthetics addressed other forms of diastemas in the anterior region. Frush and Fisher ${ }^{9}$ recommended avoiding midline diastema and preferred distributing the space symmetrically on both sides of the midline. Lombardi ${ }^{10}$ also considered midline diastema as unsightly and recommended distributing the space between the two lateral incisors, with a slight midline diastema, and two wider symmetrical diastemas between the central and lateral incisors. Simian ${ }^{11}$ diastemas are symmetrical diastemas between the lateral incisor and canine.

The need for treatment is mainly attributed to esthetic and psychological reasons, rather than functional ones. Patient's perceptions and expectations regarding their appearance play a significant role in treatment planning. A study by Kumar et al $^{12}$ has shown that threshold for unattractiveness for midline diastema was found to be less in orthodontists and dentists compared with laypersons. Facial esthetic perception differs among individuals and is often influenced by their own experiences and society and culture. What is unacceptable to professionals may be acceptable to layman. It is important for the dentist to know the esthetic perception of the various forms of diastemas in the esthetic zone.

The objectives of this study were to compare the smile attractiveness and perceptions of dental students and laypersons toward various anterior diastemas and to establish the most unattractive diastema among all.

\section{Materials and Methods}

The following descriptive cross-sectional study was conducted from September 2018 to November 2018 on 50 undergraduate dental students of Bhojia Dental College and 50 laypersons (-Table 1), which included nursing students of Bhojia Nursing College, Bhud, District Solan, Himachal Pradesh, India. Only those students were included in the study who fulfilled the following criteria.

\section{Inclusion Criteria}

1. Undergraduate dental students (4th year and intern) who have knowledge of diastema.

2. No gender specification.

3. Voluntary participation of candidates.

\section{Exclusion Criteria}

1. Any orthodontist, prosthodontist or any other dental specialization.

\section{Sample Definition}

The sample was divided into two groups on the basis of their perceptions of midline diastema. The first group consisted of 4th and 5th year dental undergraduates who had some knowledge of midline diastema and the second group, of nursing students with no such knowledge.

\section{Methodology}

Two questionnaires were prepared for the present study. The first questionnaire ( - Table 2 ) required the personal details of the subjects.

The second questionnaire (-Fig. 1) consisted of the edited frontal photograph of a patient who had reported to the Department of Orthodontics and Dentofacial Orthopaedics with the chief complaint of midline diastema. The patient had signed informed written consent for this purpose. A photograph of midline diastema was kept and then the photograph was edited using Adobe photoshop CS6 version, to create various types of diastemas that were then arranged according to types given by Frush and Fisher, Lombardi, and Simian. They were midline diastema (-Fig. 1A), asymmetrical diastemas between the canine and the lateral incisor on one side and between the lateral and central incisors on the other side (Frush and Fisher diastema [-Fig. 1B]), slight midline diastema and two larger diastemas between the central and lateral incisors (Lombardi diastema [ - Fig. 1C]), and symmetrical diastemas between the lateral incisors and the canines (Simian diastema [ - Fig. 1D]). ${ }^{8-10}$

The participants were asked to rank the various midline diastema photographs using a visual analog scale (VAS) from 1 to 4 as depicted in - Table 3 .

The objectives of the study were explained to the participants and the questionnaires were distributed in one of the morning class of dental section and then on other day in nursing section by taking permission from the faculty who waited outside. A pilot study on 10 (five boys and five girls) 5th year dental undergraduates was done to establish the time for unbiased opinion and to determine reliability of the questionnaire. The participants were explained the procedure and given a total of 5 minutes to completely fill the questionnaire and rank the smile attractiveness of

Table 1 Grouping of subjects

\begin{tabular}{|l|l|}
\hline Group I $(\boldsymbol{n}=\mathbf{5 0})$ & Group II $(\boldsymbol{n}=\mathbf{5 0})$ \\
\hline Dental undergraduates & Laypersons (nursing undergraduates) \\
\hline
\end{tabular}

Table 2 First questionnaire

\begin{tabular}{|l|l|}
\hline \multicolumn{2}{|l|}{ Personal details } \\
\hline 1. & Name \\
\hline 2. & Age/Sex \\
\hline 3. & Qualification \\
\hline 4. & Knowledge of diastema \\
\hline
\end{tabular}




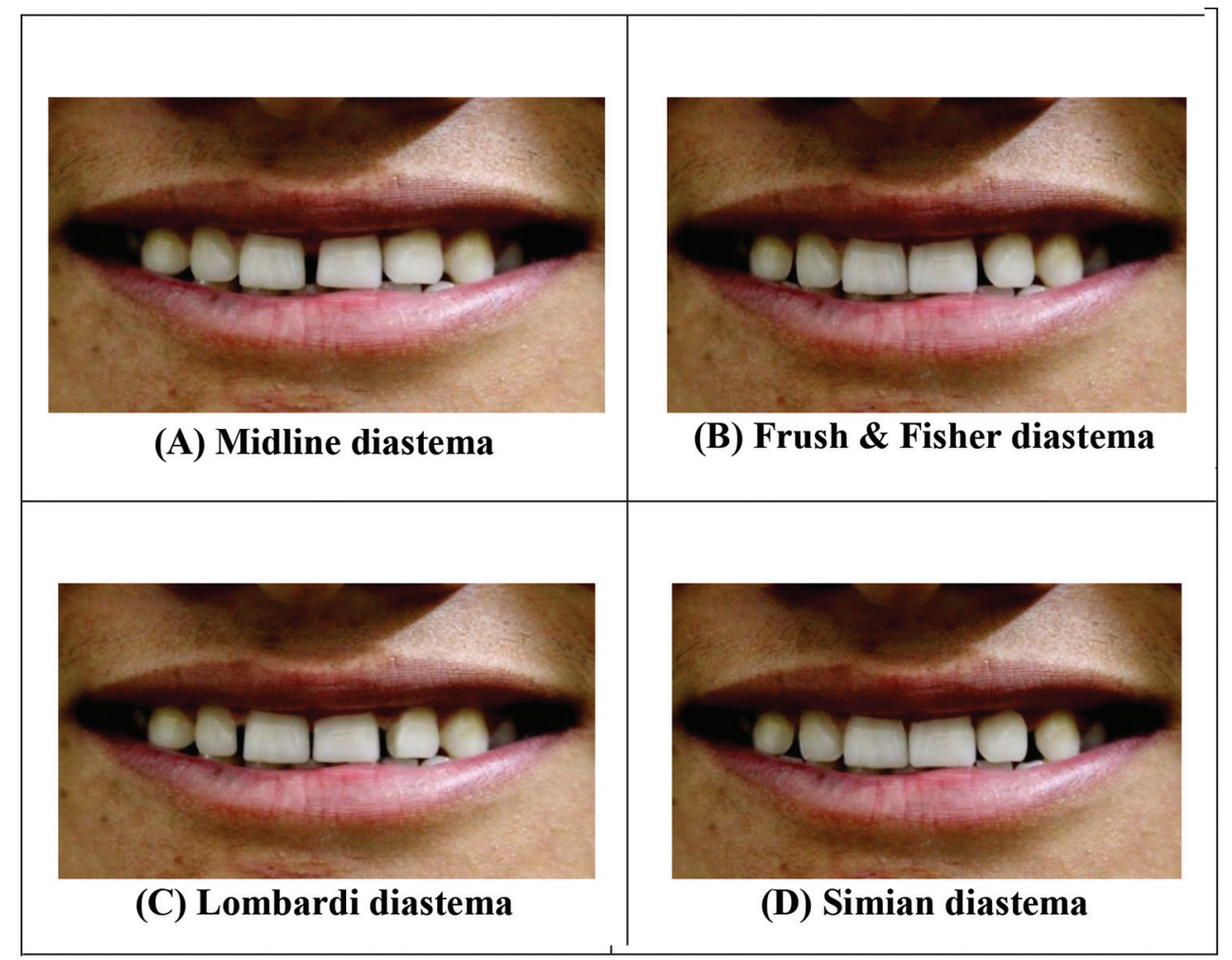

Fig. 1 Photographs of various diastemas (A-D) used for evaluation.

Table 3 Visual analog scale

\begin{tabular}{|l|l|}
\hline Rank & Perception \\
\hline 1 & Most attractive \\
\hline 2 & Attractive \\
\hline 3 & Less attractive \\
\hline 4 & Least attractive \\
\hline
\end{tabular}

the patient. The participation was voluntary and they had signed a written consent for the same.

\section{Statistical Analysis}

The data were collected and tabulated. Statistical Package for the Social Sciences (SPSS; version 16, SPSS Inc, Chicago, Illinois, United States) software was used to analyze the data. Comparison between different variables was made by chi-squared test.

\section{Results}

The students ranked midline diastema differently. On compiling the attractive and most attractive scores (i.e., VAS rank 1 and rank 2), attractiveness of diastema was calculated as below. It was shown that $0 \%$ of dental students and $44 \%$ of nursing students considered midline diastema as attractive (-Fig. 2), 50\% of dental students and 56\% of nursing students ranked Frush and Fisher as attractive (-Fig. 3), 20\% dental students and 44\% nursing students ranked Lombardi diastema as attractive (-Fig. 4), and 68\% dental students and 78\% nursing students ranked Simian diastema as attractive (-Fig. 5). Smile attractiveness from the most attractive to least attractive was Simian $>$ Frush and Fisher > Lombardi > midline diastema among both dental and nursing students as shown in (-Table 4). Regarding the impact of the anterior diastema on the student's social life, almost $45 \%$ of the students said they were not affected by its presence. Unpleasant feelings when smiling were associated with the presence of diastema among almost 55\% and they thought it has an effect on their social life (-Fig. 6 ).

\section{Discussion}

Smile plays a major role in self-perception of an individual and acts as an important element of physical attractiveness and facial expression. Spacing localized or generalized is a major esthetic problem for many patients. Diastema affects social life as majority of students with midline diastema feel shy while smiling. ${ }^{13}$ These results are similar to a study done in European adults showing that patients having spaces in their teeth were found to be less successful. ${ }^{14}$ In other cases, diastema is considered as a sign of beauty and these individuals have self-satisfaction about their smiles. The present study was based on evaluating various spaces in the esthetic zone and establishing the most unattractive diastema among all.

For this, two questionnaires were prepared. The first part contained questions about name, age, gender, qualification, and knowledge of diastema, while the second questionnaire consisted of four photographs that were created by Adobe photoediting software CS6 and then arranged according to 


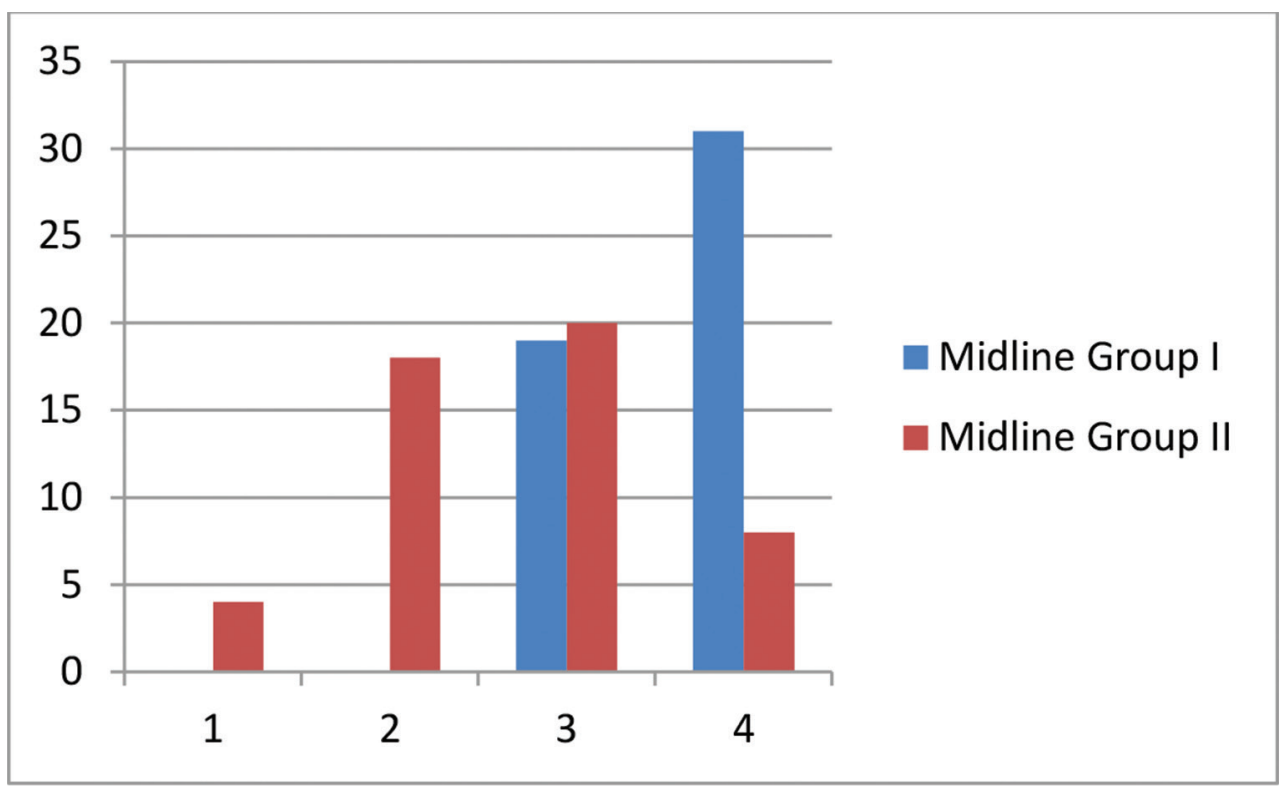

Fig. 2 Comparison of visual analog scale ranking of midline diastema between groups I and II.

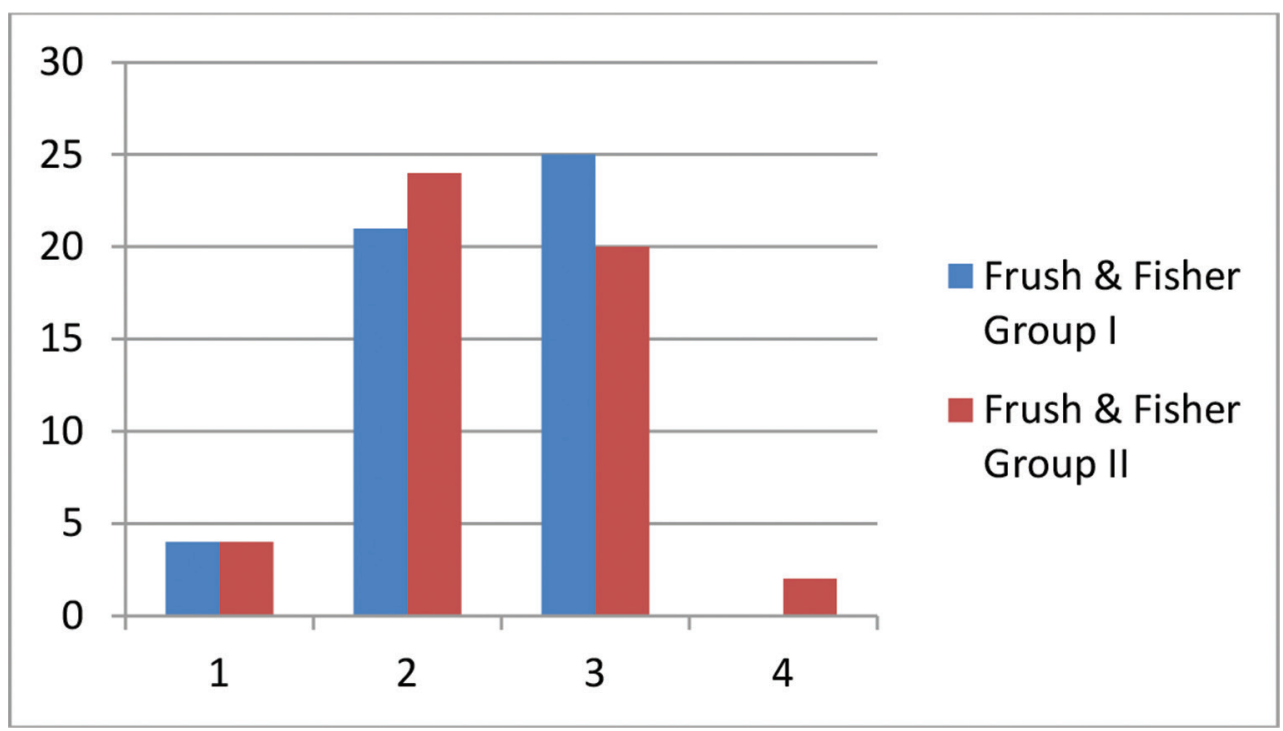

Fig. 3 Comparison of visual analog scale ranking of Frush and Fisher diastema between groups I and II.

diastemas used in the previous studies of Frush and Fisher, Lombardi, and Simian.

The students highly disagreed that midline diastema is a sign of beauty, which explains that they were more self-satisfied with their smile appearance and for them their smile is ideal than those who had diastema. However, the overall diastema perception showed a difference of only $5 \%$ where $45 \%$ students considered it attractive and $55 \%$ considered diastemas as unattractive. It supports the study by Kokich et $\mathrm{al}^{15}$ and Thomas et $\mathrm{al}^{16}$ where the perceptions of dentists and laypersons toward diastema did not differ significantly.

The present study aimed to determine the perceptions of diastema by both dental and nursing students. As dental students had knowledge of midline diastema so they ranked it as unattractive and hence showed significant value when compared with nursing students who found it attractive. Other than this, there was no significant difference in other classifications when compared between dental and nursing students. Both dental and nursing students ranked Simian diastema as most attractive followed by Frush and Fisher, Lombardi, and, finally, midline diastema.

Simian and Frush and Fisher diastemas were ranked as the two most attractive smiles, where both of them have bilateral gaps; whereas midline and Lombardi diastemas were ranked as the two least attractive diastemas as both of them have a gap in the midline. This indicates that the midline diastema has an important role in smile attractiveness.

Midline diastema was the gap with the most negative effect on smile. The present study shows similarity to the results of a study done by Noureddine et $\mathrm{al},{ }^{11}$ where midline diastema was the gap with the most negative effect on smile. 


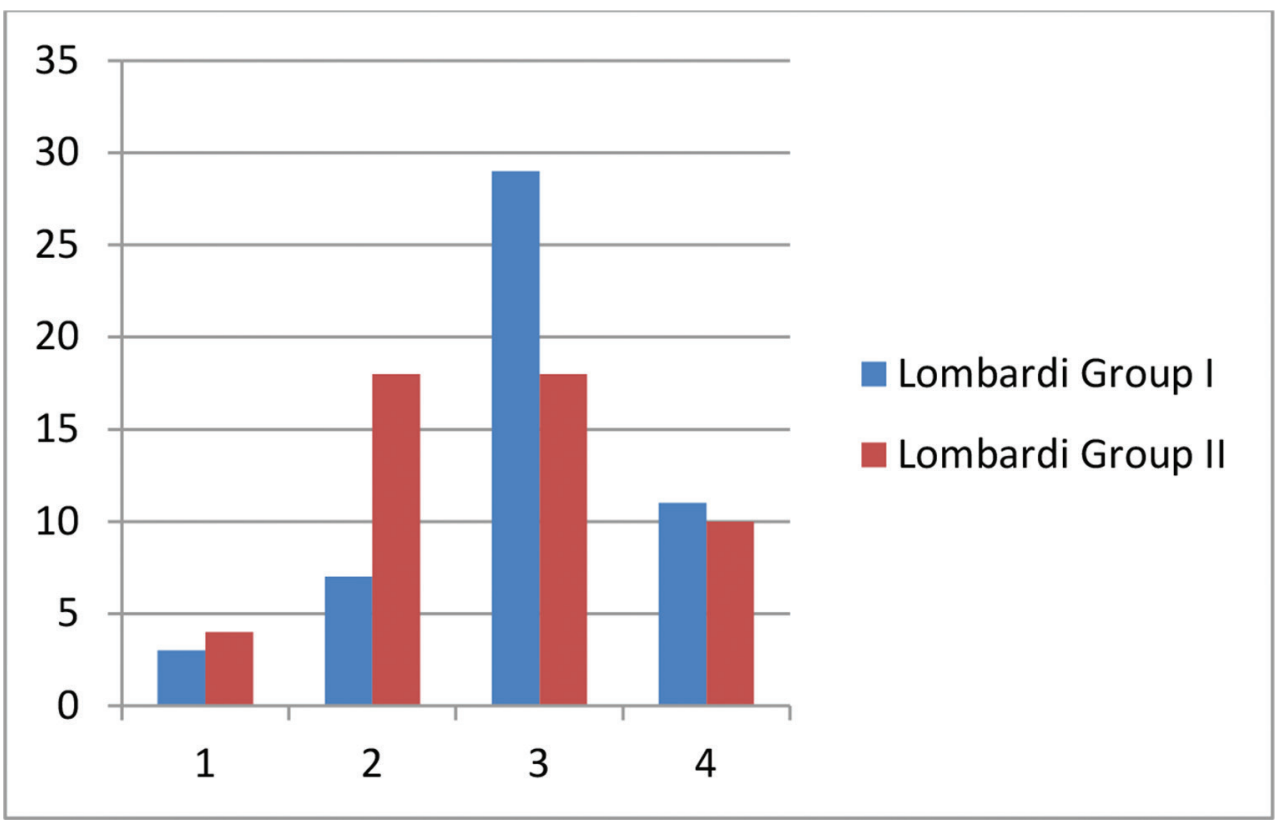

Fig. 4 Comparison of visual analog scale ranking of Lombardi diastema between groups I and II.

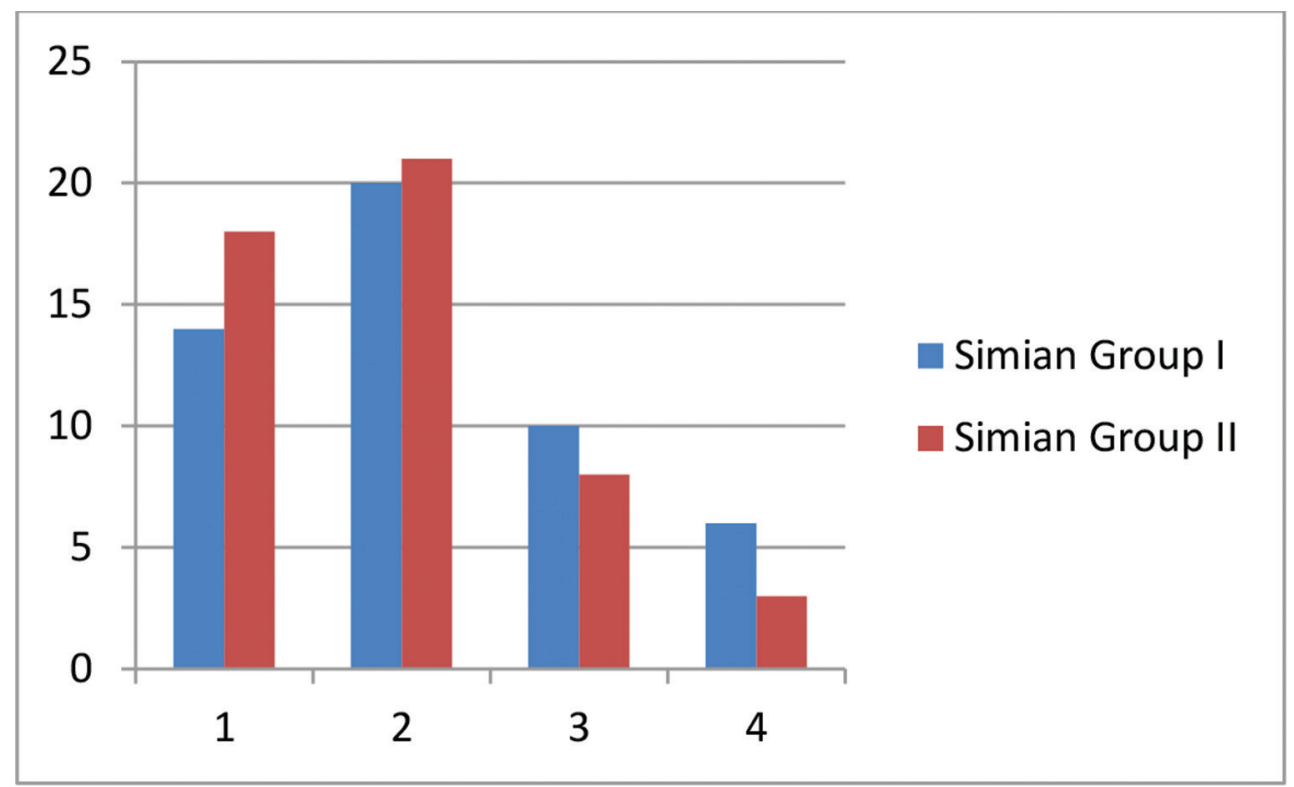

Fig. 5 Comparison of visual analog scale ranking of Simian diastema between groups I and II.

This study shows differences to the study done by Umanah et $\mathrm{al}^{17}{ }^{17}$ where maxillary midline diastema was desired and considered an attractive feature.

A small midline diastema was not rated as unattractive by any group in a study done by Thomas et $\mathrm{al}^{16}$ and Swetha and Christine, ${ }^{18}$ in contrast to our study where Lombardi diastema, which has a small midline diastema, was rated as the least attractive. Similar results were obtained by others, which suggest that there is a relation between the size of diastema and smile attractiveness.

This study shows some limitations as the width of diastema was not measured while creating spaces. Also, due to absence of boys in nursing wing, the gender was not considered in the study, which, according to our pilot study, did not show any significant difference.

\section{Conclusion}

The perceptions of smile according to different diastemas from the most to the least attractive were as follows: Simian diastema $>$ Frush and Fisher diastema $>$ Lombardi $>$ midline diastema. Midline diastema was the gap with the most negative influence on smile. No significant difference was seen in the perceptions of various anterior diastemas by dental undergraduates and laypersons, other than midline diastema. The location and width of diastema had an effect on smile attractiveness, which affects 
Table 4 Comparison of perception toward anterior diastemas between groups I and II

\begin{tabular}{|c|c|c|c|c|c|c|}
\hline $\begin{array}{l}\text { Types of anterior } \\
\text { diastemas }\end{array}$ & Group & $\begin{array}{l}\text { Most attractive, } \\
n(\%)\end{array}$ & $\begin{array}{l}\text { Attractive, } \\
n(\%)\end{array}$ & $\begin{array}{l}\text { Less attractive, } \\
n(\%)\end{array}$ & $\begin{array}{l}\text { Least attractive, } \\
n(\%)\end{array}$ & $p$-Value \\
\hline \multirow[t]{3}{*}{ Midline } & Group-I & 0 & 0 & 19 & 31 & \multirow[t]{3}{*}{$0.00^{\mathrm{a}}$} \\
\hline & Group-II & 4 & 18 & 20 & 8 & \\
\hline & Total & 4 & 18 & 39 & 39 & \\
\hline \multirow[t]{3}{*}{ Frush and Fisher } & Group-I & 4 & 21 & 25 & 0 & \multirow[t]{3}{*}{0.43} \\
\hline & Group-II & 4 & 24 & 20 & 2 & \\
\hline & Total & 8 & 45 & 45 & 2 & \\
\hline \multirow[t]{3}{*}{ Lombardi } & Group-I & 3 & 7 & 29 & 11 & \multirow[t]{3}{*}{0.88} \\
\hline & Group-II & 4 & 18 & 18 & 10 & \\
\hline & Total & 7 & 25 & 47 & 21 & \\
\hline \multirow[t]{3}{*}{ Simian } & Group-I & 14 & 20 & 10 & 6 & \multirow[t]{3}{*}{0.55} \\
\hline & Group-II & 18 & 21 & 8 & 3 & \\
\hline & Total & 32 & 41 & 18 & 9 & \\
\hline
\end{tabular}

aSignificance level is less than 0.001 .

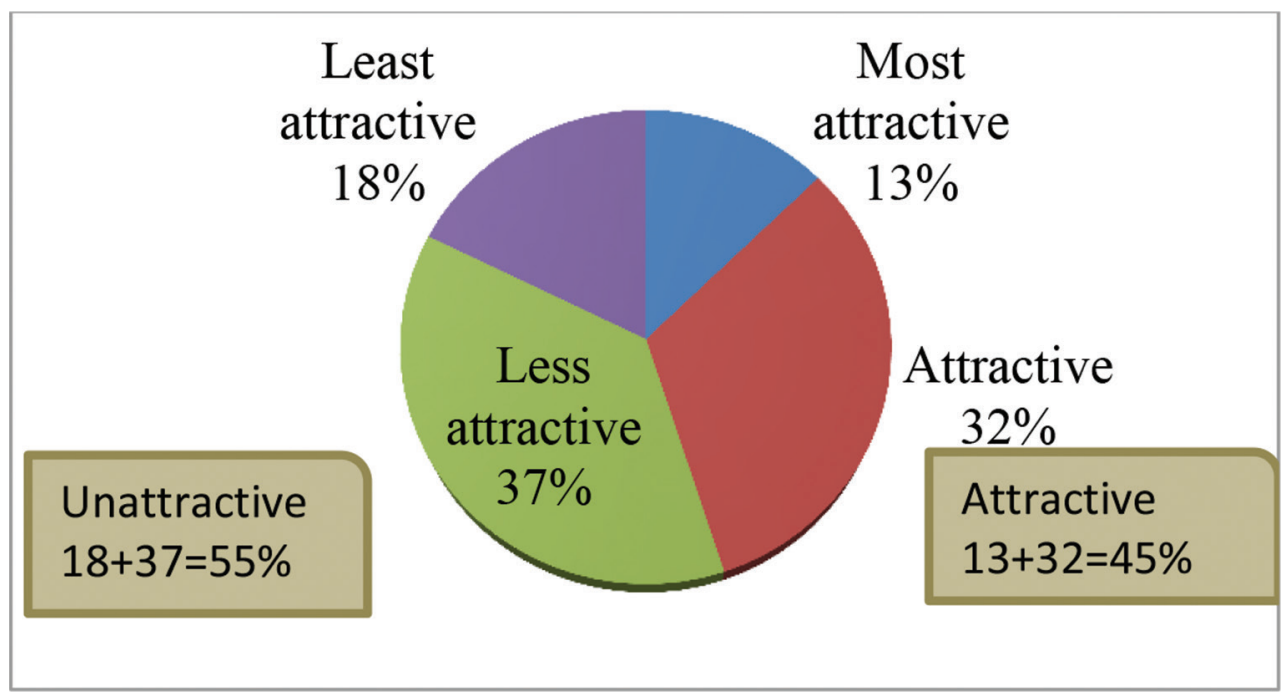

Fig. 6 The overall perception of students toward all diastemas.

patient's social life. Dentists should be aware while treating a smile with excessive anterior spacing and always bear in mind that the perception of smile attractiveness varies according to the individual's age, gender, and culture.

\section{Conflict of Interest}

None declared.

\section{References}

1 Thompson LA, Malmberg J, Goodell NK, Boring RL. The distribution of attention across a talker's face. Discourse Process 2004;38:145-168

2 Rodrigues Cde D, Magnani R, Machado MS, Oliveira OB. The perception of smile attractiveness. Angle Orthod 2009;79(4):634-639

3 Broadbent B. Ontogenic development of occlusion. Angle Orthod 1941;11:223-241

4 Dunn WJ, Murchison DF, Broome JC. Esthetics: patients' perceptions of dental attractiveness. J Prosthodont 1996;5(3):166-171
5 Baum AT. The midline diastema. J Oral Med 1966;21(1):30-39

6 Nagalakshmi S, Sathish R, Priya K, Dhayanithi D. Changes in quality of life during orthodontic correction of midline diastema. J Pharm Bioallied Sci 2014;6(Suppl 1):S162-S164

7 Nainar SM, Gnanasundaram N. Incidence and etiology of midline diastema in a population in south India (Madras). Angle Orthod 1989;59(4):277-282

8 Huang WJ, Creath CJ. The midline diastema: a review of its etiology and treatment. Pediatr Dent 1995;17(3):171-179

9 Frush JP, Fisher RD. The dynesthetic interpretation of the dentogenic concept. J Prosthet Dent 1958;8:558-581

10 Lombardi RE. A method for the classification of errors in dental esthetics. J Prosthet Dent 1974;32(5):501-513

11 Noureddine A, Fron Chabouis H, Parenton S, Lasserre JF. Laypersons' esthetic perception of various computer-generated diastemas: a pilot study. J Prosthet Dent 2014;112(4):914-920

12 Kumar S, Gandhi S, Valiathan A. Perception of smile esthetics among Indian dental professionals and laypersons. Indian J Dent Res 2012;23(2):295

13 Houacine A, Awooda EM. Perception of smile attractiveness toward various forms of anterior diastemas among 
undergraduate dental and non-dental students: a questionnaire-based study. Int J Orthod Rehab 2017;8(3):96-100

14 Kaya D, Taner TU. Management of an adult with spaced dentition, class III malocclusion and open-bite tendency. Eur J Dent 2011;5(1):121-129

15 Kokich VO, Kokich VG, Kiyak HA. Perceptions of dental professionals and laypersons to altered dental esthetics: asymmetric and symmetric situations. Am J Orthod Dentofacial Orthop 2006;130(2):141-151

16 Thomas M, Reddy R, Reddy BJ. Perception differences of altered dental esthetics by dental professionals and laypersons. Indian J Dent Res 2011;22(2):242-247
17 Umanah A, Omogbai AA, Osagbemiro B. Prevalence of artificially created maxillary midline diastema and its complications in a selected Nigerian population. Afr Health Sci 2015;15(1):226-232

18 Swetha S, Christine S. Comparison of perception of smile by orthodontists and other specialty dentists: a questionnaire study. Int J Orthod Rehabil 2016;7:92-96 